Chirurg 2019 $\cdot 90: 325$

https://doi.org/10.1007/s00104-019-0946-9

Online publiziert: 25. März 2019

(c) Springer Medizin Verlag GmbH, ein Teil von Springer Nature 2019

\section{Originalpublikation}

Margonis GA, Sergentanis TN, NtanasisStathopoulos I et al (2018) Impact of surgical margin width on recurrence and overall survival following $\mathrm{r} 0$ hepatic-resection of colorectal metastases: a systematic review and meta-analysis. Ann Surg. 267(6):1047-1055. https://doi.org/10.1097/ SLA.0000000000002552

Hintergrund. Es gibt viele Faktoren, welche die Prognose nach R0-Resektion kolorektaler Lebermetastasen beeinflussen, von denen einzig der Abstand zum Resektionsrand durch den Chirurgen teilweise kontrolliert werden kann, um optimale Ergebnisse zu erzielen. Obwohl ein tumorfreier Resektionsrand für die Prognose von Patienten mit kolorektalen Lebermetastasen von größter Bedeutung ist, bleibt die optimale Breite des Resektionsrandes umstritten. In dieser Arbeit wurde untersucht, ob sich der tumorfreie Abstand zum Resektionsrand bei einer R0-Leberresektion kolorektaler Metastasen auf das Überleben auswirkt.

Methodik. Es wurden Studien aus den Datenbanken Medline, Cochrane und EMBASE zusammengetragen, in denen der Zusammenhang zwischen dem Abstand zum Resektionsrand nach R0Resektion kolorektaler Lebermetastasen und dem „overall survival“ (OS) sowie dem „disease-free survival“ (DFS) untersucht wurden. Studien wurden eingeschlossen, wenn sie folgende Kriterien erfüllten: bei den eingeschlossenen Patienten wurden kolorektale Lebermetastasen diagnostiziert und diese galten als resektabel; es wurden R0-Resektionen durchgeführt und der tumorfreie Abstand zum Resektionsrand dokumentiert. Untergruppen von Patienten

\title{
Abstand zum Resektionsrand bei R0-Leberresektionen
}

mit verschiedenen Resektionsabständen wurden verglichen $(<1 \mathrm{~mm},<1 \mathrm{~cm}$, $>1 \mathrm{~cm}$ ) und dabei OS und DFS nach 1, 3, 5 und 10 Jahren festgehalten. Für die Kalkulation des gepoolten relativen Risikos (RR) mit einem $95 \%$-Konfidenzintervall (95\%-CI) wurden lineare Paneldatenmodelle verwendet. Es wurden separate Analysen nach Zeitpunkt (1-, 3-, 5- und 10-Jahre-OS und DFS) und in Abhängigkeit der Breite des Resektionsrandes ( $<1 \mathrm{~cm}$ vs. $>1 \mathrm{~cm}$ und $<1 \mathrm{~mm}$ vs. $>1 \mathrm{~mm}$ ) durchgeführt.

Ergebnisse. Insgesamt 623 Abstracts wurden durch den Suchalgorithmus gefunden, ausgeschlossen wurden: 501 irrelevante Abstracts, 4 Abstracts zeigten Überlappungen, 70 Abstracts aufgrund mangelnder Daten und 22 Reviews. 34 Studien, welche eine Kohorte von insgesamt 11.147 Leberresektionen widerspiegelten, wurden in die Untersuchungen eingeschlossen. Ein tumorfreier Sicherheitsabstand zum Resektionsrand von über $1 \mathrm{~cm}$ verglichen zu unter $1 \mathrm{~cm}$ $(>1 \mathrm{~cm}$ vs. $<1 \mathrm{~cm})$ war signifikant mit einem besseren OS nach 3 Jahren (gepooltes RR 1/4 0,86; $95 \%$-CI: 0,79-0,95), nach 5 Jahren (gepooltes RR 1/4 0,91; $95 \%$-CI 0,85-0,97) und nach 10 Jahren (gepooltes RR 1/4 0,94; 0,88-1,00) verbunden. Ein Abstand von $>1 \mathrm{~cm}$ wirkte sich ebenfalls positiv auf das DFS nach 3, 5 und 10 Jahren aus. Es zeigte sich außerdem, dass ein Abstand von $>1 \mathrm{~mm}$ im Vergleich $\mathrm{zu}<1 \mathrm{~mm}$ zu allen Nachbeobachtungszeitpunkten mit einem signifikant besseren OS assoziiert war. In der Metaregressionsanalyse zeigten sich keine signifikanten Zusatzeffekte in den untersuchten Studienmerkmalen durch: die Verabreichung einer neoadjuvanten oder adjuvanten Therapie, das durchschnittliche Patientenalter, die Geschlechterverteilung, das Publikationsjahr, Anteil synchroner hepatischer Metastasierung, primäre Tumorgröße oder durchschnittliche Anzahl an Lebermetastasen.

\section{Zusammenfassung}

Die Ergebnisse zeigen, dass ein tumorfreier Resektionsrand von mindestens $1 \mathrm{~mm}$ zwar mit einer verbesserten Prognose verbunden ist, aber ein Abstand von $>1 \mathrm{~cm}$ zu noch besseren onkologischen Ergebnissen führen kann und in Hinsicht auf das OS signifikant unabhängig von der angewendeten R0/R1-Definition ist. Dennoch variieren die Ergebnisse in der Studienpopulation in Abhängigkeit von verschiedenen Faktoren, wie z. B. der Tumorbiologie. Auch durch das Verwenden verschiedener Methoden zur Leberresektion wie z. B. CUSA ${ }^{\circledR}$ oder TissueLink $^{\text {ma }}$ (Salient Surgical Technologies, Portsmouth, NH, USA) wird die Vergleichbarkeit der Studien limitiert, da der messbare Resektionsabstand durch die Aspiration von Gewebe im pathologischen Präparat durch diese verfälscht wird.

Insgesamt bestätigen die Daten, dass ein Sicherheitsabstand von $>1 \mathrm{~cm}$ eingehalten werden sollte, wenn dies möglich ist.

\section{Korrespondenzadresse}

\section{Rohland}

Klinik für Allgemein-, Viszeral- und Gefäßchirurgie, Universitätsklinikum Jena Am Klinikum 1, 07747 Jena, Deutschland Oliver.Rohland@med.uni-jena.de

Interessenkonflikt. O. Rohland, M. Ardelt und U. Settmacher geben an, dass kein Interessenkonflikt besteht. 